\title{
UNTYPING TYPED ALGEBRAS AND COLOURING CYCLIC LINEAR LOGIC*
}

\author{
DAMIEN POUS
}

CNRS (LIG, UMR 5217, Grenoble)

e-mail address: Damien.Pous@ens-lyon.fr

\begin{abstract}
We prove "untyping" theorems: in some typed theories (semirings, Kleene algebras, residuated lattices, involutive residuated lattices), typed equations can be derived from the underlying untyped equations. As a consequence, the corresponding untyped decision procedures can be extended for free to the typed settings. Some of these theorems are obtained via a detour through fragments of cyclic linear logic, and give rise to a substantial optimisation of standard proof search algorithms.
\end{abstract}

\section{INTRODUCTION}

Motivations. The literature contains many decidability or complexity results for various algebraic structures. Some of these structures (rings, Kleene algebras [22], residuated lattices [31]) can be generalised to typed structures, where the elements come with a domain and a codomain, and where operations are defined only when these domains and codomains agree according to some simple rules. Although such typed structures are frequently encountered in practice (e.g., rectangular matrices, heterogeneous binary relations, or more generally, categories), there are apparently no proper tools to easily reason about these.

This is notably problematic in proof assistants, where powerful decision procedures are required to let the user focus on difficult reasoning steps by leaving administrative details to the computer. Indeed, although some important theories can be decided automatically in Coq or HOL (e.g., Presburger arithmetic [29], elementary real algebra [15], rings [14]), there are no high-level tools to reason about heterogeneous relations or rectangular matrices.

In this paper, we show how to extend the standard decision procedures from the untyped structures to the corresponding typed structures. In particular, we make it possible to use standard tools to reason about rectangular matrices or heterogeneous relations, without bothering about types (i.e., matrix dimensions or domain/codomain information). The approach we propose is depicted below: we study "untyping" theorems that allow one to

1998 ACM Subject Classification: F.4.1, F.4.3.

Key words and phrases: involutive residuated lattices, cyclic linear logic, Kleene algebra, typed algebra, decision procedures, sequent calculus, proof search.

* Extended version of the abstract that appeared in Proc. CSL'10 [34].

Partially funded by the French projects "Choco", ANR-07-BLAN-0324 and "PiCoq", ANR-10-BLAN-0305.

LOGICAL METHODS IN COMPUTER SCIENCE

DOI:10.2168/LMCS-8 (2:12) 2012

(C) D. Pous (c) Creative Commons 
prove typed equations as follows: 1) erase type informations, 2) prove the equation using standard, untyped, decision procedures, and 3) derive a typed proof from the untyped one.

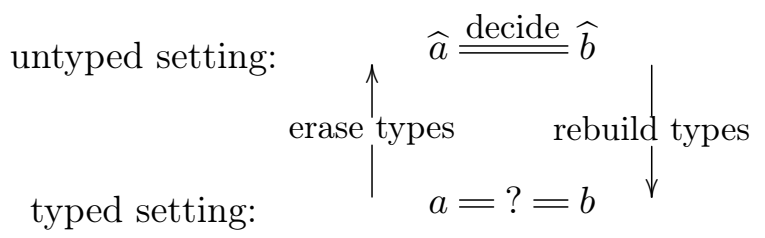

Besides the theoretical aspects, an important motivation behind this work comes from a Coq library [5] in which we developed efficient tactics for partial axiomatisations of relations: the ideas presented here were used and integrated in this library to extend our tactics to typed structures, for free.

Overview. We shall mainly focus on the two algebraic structures we mentioned above, since they raise different problems and illustrate several aspects of these untyping theorems: Kleene algebras [21] and residuated lattices [19].

- The case of Kleene algebras is the simplest one. The main difficulty comes from the annihilating element (0): its polymorphic typing rule requires us to show that equational proofs can be factorised so as to use the annihilation laws at first, and then reason using the other axioms.

- The case of residuated structures is more involved: due to the particular form of axioms about residuals, we cannot rely on standard equational axiomatisations of these structures. Instead, we need to exploit an equivalent cut-free sequent proof system (first proposed by Ono and Komori [31]), and to notice that this proof system corresponds to the intuitionistic fragment of cyclic linear logic [40. The latter logic is much more concise and the corresponding proof nets are easier to reason about, so that we obtain the untyping theorem in this setting. We finally port the result back to residuated lattices by standard means.

The above sequent proof systems have the sub-formula property, so that they yield decision procedures, using proof search algorithms. As an unexpected application, we show that the untyping theorem makes it possible to improve these algorithms by reducing the set of proofs that have to be explored.

Outline. We introduce our notations and make the notion of typed structure precise in $\$ 1$. We study Kleene algebras and residuated lattices in $\$ 2$ and $\$ 3$, respectively. The optimisation of proof search is analysed in $\$ 4$, we conclude with related work, and directions for future work in $\$ 5$.

\section{Notation, typed Structures}

Let $\mathcal{X}$ be an arbitrary set of variables, ranged over using letters $x, y$. Given a signature $\Sigma$, we let $a, b, c$ range over the set $T(\Sigma+\mathcal{X})$ of terms with variables. Given a set $\mathcal{T}$ of objects (ranged over using letters $n, m, p, q)$, a type is a pair $(n, m)$ of objects (which we denote by $n \rightarrow m$, following categorical notation), a type environment $\Gamma: \mathcal{X} \rightarrow \mathcal{T}^{2}$ is a function from variables to types, and we will define type judgements of the form $\Gamma \vdash a: n \rightarrow m$, to be read "in environment $\Gamma$, term $a$ has type $n \rightarrow m$, or, equivalently, $a$ is a morphism from $n$ to $m$ ". 
By $\Gamma \vdash a, b: n \rightarrow m$, we mean that both $a$ and $b$ have type $n \rightarrow m$; type judgements will include the following rule for variables:

$$
\frac{\Gamma(x)=(n, m)}{\Gamma \vdash x: n \rightarrow m} \mathrm{TV}
$$

Similarly, we will define typed equality judgements of the form $\Gamma \vdash a=b: n \rightarrow m$ : "in environment $\Gamma$, terms $a$ and $b$ are equal, at type $n \rightarrow m$ ". Equality judgements will generally include the following rules, so as to obtain an equivalence relation at each type:

$$
\begin{array}{lll} 
& \Gamma \vdash a=b: n \rightarrow m \\
\frac{\Gamma(x)=(n, m)}{\Gamma \vdash x=x: n \rightarrow m} \mathrm{~V} & \frac{\Gamma \vdash b=c: n \rightarrow m}{\Gamma \vdash a}=c: n \rightarrow m & \\
\mathrm{~T} & \frac{\Gamma \vdash a=b: n \rightarrow m}{\Gamma \vdash b=a: n \rightarrow m} \mathrm{~S}
\end{array}
$$

By taking the singleton set as set of objects $(\mathcal{T}=\{\emptyset\})$, we recover standard, untyped structures: the only typing environment is $\widehat{\emptyset}: x \mapsto(\emptyset, \emptyset)$, and types become uninformative (this corresponds to working in a one-object category; all operations are total functions). To alleviate notations, since the typing environment will always be either $\widehat{\emptyset}$ or an abstract constant value $\Gamma$, we shall leave it implicit in type and equality judgements, by relying on the absence or the presence of types to indicate which one to use. For example, we shall write $\vdash a=b: n \rightarrow m$ for $\Gamma \vdash a=b: n \rightarrow m$, while $\vdash a=b$ will denote the judgement $\widehat{\emptyset} \vdash a=b: \emptyset \rightarrow \emptyset$.

The question we study in this paper is the following one: given a signature and a set of inference rules defining a type judgement and an equality judgement, does the implication below hold, for all $a, b, n, m$ ?

$$
\left\{\begin{array}{l}
\vdash a, b: n \rightarrow m \\
\vdash a=b
\end{array} \quad \text { entails } \quad \vdash a=b: n \rightarrow m .\right.
$$

In other words, in order to prove an equality in a typed structure, is it safe to remove all type annotations, so as to work in the untyped underlying structure?

\section{KLEEnE ALGEBRAS}

We study the case of residuated lattices in $\$ 3$, here we focus on Kleene algebras. In order to illustrate our methodology, we actually give the proof in three steps, by considering two intermediate algebraic structures: monoids and semirings. The former admit a rather simple and direct proof, while the latter are sufficient to expose concisely the main difficulty in handling Kleene algebras. 


\subsection{Monoids.}

Definition 2.1. Typed monoids are defined by the signature $\left\{\cdot_{2}, 1_{0}\right\}$, together with the following inference rules, in addition to the rules from $\$ 1$

$$
\begin{aligned}
& \frac{\vdash a: n \rightarrow m \quad \vdash b: m \rightarrow p}{\vdash 1: n \rightarrow n} \text { Tо } \text { TD } \quad \frac{\vdash a \cdot b: n \rightarrow p}{\vdash 1=1: n \rightarrow n} \text { O } \\
& \frac{\vdash a=a^{\prime}: n \rightarrow m \quad \vdash b=b^{\prime}: m \rightarrow p}{\vdash a \cdot b=a^{\prime} \cdot b^{\prime}: n \rightarrow p} \mathrm{D} \quad \frac{\vdash a: n \rightarrow m}{\vdash 1 \cdot a=a: n \rightarrow m} \text { OD } \\
& \frac{\vdash a: n \rightarrow m \quad \vdash b: m \rightarrow p \quad \vdash c: p \rightarrow q}{\vdash(a \cdot b) \cdot c=a \cdot(b \cdot c): n \rightarrow q} \mathrm{DA} \quad \frac{\vdash a: n \rightarrow m}{\vdash a \cdot 1=a: n \rightarrow m} \text { DO }
\end{aligned}
$$

In other words, typed monoids are just categories: 1 and $\cdot$ correspond to identities and composition. Rules (O) and (D) ensure that equality is reflexive at each type (point $(i)$ below) and preserved by composition. As expected, equalities relate correctly typed terms only (ii):

Lemma 2.2. (i) If $\vdash a: n \rightarrow m$, then $\vdash a=a: n \rightarrow m$.

(ii) If $\vdash a=b: n \rightarrow m$, then $\vdash a, b: n \rightarrow m$.

Moreover, in this setting, type judgements enjoy some form of injectivity (types are not uniquely determined due to the unit (1), which is typed in a polymorphic way):

Lemma 2.3. If $\vdash a: n \rightarrow m$ and $\vdash a: n^{\prime} \rightarrow m^{\prime}$, then we have $n=n^{\prime}$ iff $m=m^{\prime}$.

We need another lemma to obtain the untyping theorem: all terms related by the untyped equality admit the same type derivations.

Lemma 2.4. If $\vdash a=b$; then for all $n, m$, we have $\vdash a: n \rightarrow m$ iff $\vdash b: n \rightarrow m$.

Theorem 2.5. If $\vdash a=b$ and $\vdash a, b: n \rightarrow m$, then $\vdash a=b: n \rightarrow m$.

Proof. We reason by induction on the derivation $\vdash a=b$; the interesting cases are the following ones:

- the last rule used is the transitivity rule ( $\mathrm{T})$ : we have $\vdash a=b, \vdash b=c, \vdash a, c: n \rightarrow m$, and we need to show that $\vdash a=c: n \rightarrow m$. By Lemma 2.4. we have $\vdash b: n \rightarrow m$, so that by the induction hypotheses, we get $\vdash a=b: n \rightarrow m$ and $\vdash b=c: n \rightarrow m$, and we can apply rule $(\mathrm{T})$.

- the last rule used is the compatibility of $\cdot(\mathrm{D})$ : we have $\vdash a=a^{\prime}, \vdash b=b^{\prime}, \vdash a \cdot b, a^{\prime} \cdot b^{\prime}$ : $n \rightarrow m$, and we need to show that $\vdash a \cdot b=a^{\prime} \cdot b^{\prime}: n \rightarrow m$. By case analysis on the typing judgements, we deduce that $\vdash a: n \rightarrow p, \vdash b: p \rightarrow m, \vdash a^{\prime}: n \rightarrow q, \vdash b^{\prime}: q \rightarrow m$, for some $p, q$. Thanks to Lemmas 2.3 and 2.4. we have $p=q$, so that we can conclude using the induction hypotheses $\left(\vdash a=a^{\prime}: n \rightarrow p\right.$ and $\left.\vdash b=b^{\prime}: p \rightarrow m\right)$, and rule (D).

Note that the converse of Theorem 2.5 ( $\vdash a=b: n \rightarrow m$ entails $\vdash a=b)$ is straightforward, so that we actually have an equivalence. 


\subsection{Non-commutative semirings.}

Definition 2.6. Typed semirings are defined by the signature $\left\{\cdot_{2},+_{2}, 1_{0}, 0_{0}\right\}$, together with the following rules, in addition to the rules from Def. 2.1 and $\$ 1$.

$$
\begin{gathered}
F_{\vdash 0: n \rightarrow m} \mathrm{Tz} \quad \frac{\vdash a, b: n \rightarrow m}{\vdash a+b: n \rightarrow m} \mathrm{TP} \quad \frac{\vdash a=a^{\prime}: n \rightarrow m \quad \vdash b=b^{\prime}: n \rightarrow m}{\vdash a+b=a^{\prime}+b^{\prime}: n \rightarrow m} \mathrm{P} \\
\frac{\vdash a: n \rightarrow m}{\vdash 0=0: n \rightarrow m} \mathrm{Z} \quad \frac{\vdash a, b: n \rightarrow m}{\vdash a+0=a: n \rightarrow m} \mathrm{PZ} \quad \frac{\vdash a+b=b+a: n \rightarrow m}{\vdash C} \mathrm{PC} \\
\frac{\vdash a, b, c: n \rightarrow m}{\vdash(a+b)+c=a+(b+c): n \rightarrow m} \mathrm{PA} \quad \frac{\vdash a: n \rightarrow m \quad \vdash b, c: m \rightarrow p}{\vdash a \cdot(b+c)=a \cdot b+a \cdot c: n \rightarrow p} \mathrm{DP} \\
\frac{\vdash a: n \rightarrow m}{\vdash a \cdot 0=0: n \rightarrow p} \mathrm{DZ} \quad \frac{\vdash a: n \rightarrow m}{\vdash 0 \cdot a=0: p \rightarrow m} \mathrm{ZD} \quad \frac{\vdash a: n \rightarrow m \quad \vdash b, c: p \rightarrow n}{\vdash(b+c) \cdot a=b \cdot a+c \cdot a: p \rightarrow m} \mathrm{PD}
\end{gathered}
$$

In other words, typed semiring are categories enriched over a commutative monoid: each homset is equipped with a commutative monoid structure (typing rules (Tz,TP) and rules $(\mathrm{P}, \mathrm{PZ}, \mathrm{PC}, \mathrm{PA}))$, composition distributes over these monoid structures (rules (DP,DZ,PD, ZD)).

Lemma 2.2 is also valid in this setting: equality is reflexive and relates correctly typed terms only. However, due to the presence of the annihilator element (0), Lemmas 2.3 and 2.4 no longer hold: 0 has any type, and we have $\vdash x \cdot 0 \cdot x=0$ while $x \cdot 0 \cdot x$ only admits $\Gamma(x)$ as a valid type. Moreover, some valid proofs cannot be typed just by adding decorations: for example, $0=0 \cdot a \cdot a=0$ is a valid untyped proof of $0=0$; however, this proof cannot be typed if $a$ has a non-square type. Therefore, we have to adopt another strategy: we reduce the problem to the annihilator-free case, by showing that equality proofs can be factorised so as to use rules (PZ), (DZ), and ( $\mathrm{ZD})$ at first, as oriented rewriting rules.

Definition 2.7. Let $a$ be a term; we denote by $a_{\downarrow}$ the normal form of $a$, obtained with the following convergent rewriting system:

$$
\begin{array}{llll}
a+0 \rightarrow a & 0+a \rightarrow a & 0 \cdot a \rightarrow 0 & a \cdot 0 \rightarrow 0
\end{array}
$$

We say that $a$ is strict if $a_{\downarrow} \neq 0$.

This normalisation procedure preserves types and equality; moreover, on strict terms, we recover the injectivity property of types we had for monoids:

Lemma 2.8. If $\vdash a: n \rightarrow m$, then $\vdash a_{\downarrow}: n \rightarrow m$ and $\vdash a=a_{\downarrow}: n \rightarrow m$.

Lemma 2.9. For all strict terms a such that $\vdash a: n \rightarrow m$ and $\vdash a: n^{\prime} \rightarrow m^{\prime}$, we have $n=n^{\prime}$ iff $m=m^{\prime}$.

We can then define a notion of strict equality judgement, where the annihilation laws are not allowed:

Definition 2.10. We let ${ }_{-}{ }^{+}{ }_{-}=_{-}:{ }_{-} \rightarrow_{-}$denote the strict equality judgement obtained by removing rules (DZ) and $\overline{(\mathrm{ZD})}$, and replacing rules (DP) and (PD) with the 
following variants, where the factor has to be strict.

$$
\begin{aligned}
& \frac{\vdash a: n \rightarrow m \quad \vdash b, c: m \rightarrow p \quad a_{\downarrow} \neq 0}{\vdash^{+} a \cdot(b+c)=a \cdot b+a \cdot c: n \rightarrow p} \mathrm{DP}^{+} \\
& \frac{\vdash a: n \rightarrow m \quad \vdash b, c: p \rightarrow n \quad a_{\downarrow} \neq 0}{\vdash^{+}(b+c) \cdot a=b \cdot a+c \cdot a: p \rightarrow m} \mathrm{PD}^{+}
\end{aligned}
$$

Using the same methodology as previously, one easily obtain the untyping theorem for strict equality judgements.

Lemma 2.11. If $\vdash^{+} a=b$; then for all $n, m$, we have $\vdash a: n \rightarrow m$ iff $\vdash b: n \rightarrow m$.

Proposition 2.12. If $\vdash^{+} a=b$ and $\vdash a, b: n \rightarrow m$, then $\vdash^{+} a=b: n \rightarrow m$.

Note that the patched rules for distributivity, $\left(\mathrm{DP}^{+}\right)$and $\left(\mathrm{PD}^{+}\right)$are required in order to obtain Lemma 2.11: if $a$ was not required to be strict, we would have $\vdash^{+} 0 \cdot(x+y)=$ $0 \cdot x+0 \cdot y$, and the right-hand side can be typed in environment $\Gamma=\{x \mapsto(3,2), y \mapsto$ $(4,2)\}$ while the left-hand side cannot.

We now have to show that any equality proof can be factorised, so as to obtain a strict equality proof relating the corresponding normal forms:

Proposition 2.13. If $\vdash a=b$, then we have $\vdash^{+} a_{\downarrow}=b_{\downarrow}$.

Proof. We first show by induction that whenever $\vdash a=b, a$ is strict iff $b$ is strict $(\dagger)$. Then we proceed by induction on the derivation $\vdash a=b$, we detail only some cases:

(D) we have $\vdash^{+} a_{\downarrow}=a_{\downarrow}^{\prime}$ and $\vdash^{+} b_{\downarrow}=b_{\downarrow}^{\prime}$ by induction; we need to show that $\vdash^{+}$ $(a \cdot b)_{\downarrow}=\left(a^{\prime} \cdot b^{\prime}\right)_{\downarrow}$. If one of $a, a^{\prime}, b, b^{\prime}$ is not strict, then $(a \cdot b)_{\downarrow}=\left(a^{\prime} \cdot b^{\prime}\right)_{\downarrow}=0$, thanks to $(\dagger)$, so that we are done; otherwise, $(a \cdot b)_{\downarrow}=a_{\downarrow} \cdot b_{\downarrow}$, and $\left(a^{\prime} \cdot b^{\prime}\right)_{\downarrow}=a_{\downarrow}^{\prime} \cdot b_{\downarrow}^{\prime}$, so that we can apply rule $(D)$.

(DZ) trivial, since $(a \cdot 0)_{\downarrow}=0$.

(DP) we need to show that $\vdash^{+}(a \cdot(b+c))_{\downarrow}=(a \cdot b+a \cdot c)_{\downarrow}$; if one of $a, b, c$ is not strict, both sides reduce to the same term, so that we can apply Lemma $2.2(i)$ (which holds in this setting); otherwise we have $(a \cdot(b+c))_{\downarrow}=a_{\downarrow} \cdot\left(b_{\downarrow}+c_{\downarrow}\right)$ and $(a \cdot b+a \cdot c)_{\downarrow}=$ $a_{\downarrow} \cdot b_{\downarrow}+a_{\downarrow} \cdot c_{\downarrow}$, so that we can apply rule $\left(\mathrm{DP}^{+}\right)$.

We finally obtain the untyping theorem by putting all together:

Theorem 2.14. In semirings, for all $a, b, n, m$ such that $\vdash a, b: n \rightarrow m$, we have $\vdash a=b$ iff $\vdash a=b: n \rightarrow m$.

Proof. The reverse implication is straightforward; we prove the direct one. By Lemma 2.8, using the transitivity and symmetry rules, it suffices to show $\vdash a_{\downarrow}=b_{\downarrow}: n \rightarrow m$. This is clearly the case whenever $\vdash^{+} a_{\downarrow}=b_{\downarrow}: n \rightarrow m$, which follows from Props. 2.13 and 2.12 . 


\subsection{Kleene algebras.}

Kleene algebras are idempotent semirings equipped with a star operation [21]; they admit several important models, among which binary relations and regular languages (the latter is complete [25, 22]; since equality of regular languages is decidable, so is the equational theory of Kleene algebras). Like previously, we type Kleene algebras in a natural way, where star operates on "square" types: types of the form $n \rightarrow n$, i.e., square matrices or homogeneous binary relations.

Definition 2.15. We define typed Kleene algebras by the signature $\left\{\cdot_{2},+_{2}, \star_{1}, 1_{0}, 0_{0}\right\}$, together with the following rules, in addition that from Defs. 2.1 and 2.6, and \&1, and where $\vdash a \leq b: n \rightarrow m$ is an abbreviation for $\vdash a+b=b: n \rightarrow m$.

$$
\begin{aligned}
& \frac{\vdash a: n \rightarrow n}{\vdash a^{\star}: n \rightarrow n} \text { Ts } \quad \frac{\vdash a=b: n \rightarrow n}{\vdash a^{\star}=b^{\star}: n \rightarrow n} \mathrm{~s} \quad \frac{\vdash a: n \rightarrow m}{\vdash a+a=a: n \rightarrow m} \text { PI } \\
& \frac{\vdash a: n \rightarrow n}{\vdash 1+a \cdot a^{\star}=a^{\star}: n \rightarrow n} \mathrm{SP} \quad \frac{\vdash a \cdot b \leq b: n \rightarrow m}{\vdash a^{\star} \cdot b \leq b: n \rightarrow m} \mathrm{SL} \quad \frac{\vdash b \cdot a \leq b: n \rightarrow m}{\vdash b \cdot a^{\star} \leq b: n \rightarrow m} \mathrm{SR}
\end{aligned}
$$

The untyped version of this axiomatisation is that from Kozen [22]: axiom (PI) corresponds to idempotence of + , the three other rules define the star operation (we omitted the mirror image of axiom (SP), which is derivable from the other ones [5]). Note that due to rules (SL) and (SR), we are no longer in a purely equational setting; indeed, the algebra of regular events is not finitely based [36].

The proof of the untyping theorem for Kleene algebras is obtained along the lines of the proof for non-commutative semirings. We just highlight the main differences here, complete proofs are available as Coq scripts [33]. First, it is a simple exercise to check that the following lemma holds:

Lemma 2.16. For all $n$, we have $\vdash 0^{\star}=1: n \rightarrow n$.

This allows us to extend the rewriting system from Def. 2.7 with the rule $0^{\star} \rightarrow 1$, so that the annihilator can also be removed in this setting. In particular, we obtain:

Lemma 2.17. If $\vdash a: n \rightarrow m$, then $\vdash a_{\downarrow}: n \rightarrow m$ and $\vdash a=a_{\downarrow}: n \rightarrow m$.

Lemma 2.18. For all strict terms a such that $\vdash a: n \rightarrow m$ and $\vdash a: n^{\prime} \rightarrow m^{\prime}$, we have $n=n^{\prime}$ iff $m=m^{\prime}$.

Let $\vdash^{+}{ }_{-}:_{-} \rightarrow_{-}$denote the strict equality judgement obtained like previously (Def. 2.10), and where we moreover adapt rules (SL) and (SR) so that $b$ is required to be strict:

$$
\frac{\vdash^{+} a \cdot b \leq b: n \rightarrow m \quad b_{\downarrow} \neq 0}{\vdash^{+} a^{\star} \cdot b \leq b: n \rightarrow m} \mathrm{SL}^{+} \quad \frac{\vdash^{+} b \cdot a \leq b: n \rightarrow m \quad b_{\downarrow} \neq 0}{\vdash^{+} b \cdot a^{\star} \leq b: n \rightarrow m} \mathrm{SR}^{+}
$$

These patched rules $\left(\mathrm{SL}^{+}\right)$and $\left(\mathrm{SR}^{+}\right)$are required to obtain the following counterpart to Lemma 2.11. otherwise, we would have $\vdash^{+} a^{\star} \cdot 0 \leq 0$, where the right-hand side has any type while the type of the left-hand side is constrained by $a$.

Lemma 2.19. If $\vdash^{+} a=b$; then for all $n, m$, we have $\vdash a: n \rightarrow m$ iff $\vdash b: n \rightarrow m$. 
Proof. Similar to the proof of Lemma 2.11. Recall that $\vdash^{+} a \leq b$ is an abbreviation for $\vdash^{+} a+b=b$; the rule $\left(\mathrm{SL}^{+}\right)$is handled as follows. Suppose that $\vdash^{+} a^{\star} \cdot b+b=b$ was obtained using this rule:

- if $\vdash a^{\star} \cdot b+b: n \rightarrow m$, then we necessarily have $\vdash b: n \rightarrow m$;

- conversely, if $\vdash b: n \rightarrow m$ then we have $\vdash a \cdot b+b: n \rightarrow m$ by induction. Therefore, there exists $p$ such that $\vdash a: n \rightarrow p$ and $\vdash b: p \rightarrow m$. Since $b$ was required to be strict, we can use Lemma 2.18 to deduce $n=p, \vdash a: n \rightarrow n$, and finally, $\vdash a^{\star} \cdot b+b: n \rightarrow m$. Rule $\left(\mathrm{SR}^{+}\right)$is handled symmetrically, and rule (SP) is straightforward.

The untyping theorem for strict equality follows easily:

Proposition 2.20. If $\vdash^{+} a=b$ and $\vdash a, b: n \rightarrow m$, then $\vdash^{+} a=b: n \rightarrow m$.

Proof. Like for Theorem 2.5 and Prop. 2.12, we proceed by induction on the untyped derivation to add type annotations. We detail the case of rule $\left(\mathrm{SL}^{+}\right)$: suppose that $\vdash^{+} a^{\star} \cdot b \leq b$ was obtained using the untyped version of rule $\left(\mathrm{SL}^{+}\right)$, and $\vdash a^{\star} \cdot b+b, b: n \rightarrow m$. Necessarily, $\vdash a: n \rightarrow n$ and $\vdash a \cdot b+b: n \rightarrow m$, so that we have $\vdash a \cdot b \leq b: n \rightarrow m$ by induction. We conclude using the typed version of rule $\left(\mathrm{SL}^{+}\right): \vdash a^{\star} \cdot b \leq b: n \rightarrow m$.

We finally have to prove that Kleene algebra equality proofs can be factorised using the strict equality judgement:

Proposition 2.21. If $\vdash a=b$, then we have $\vdash^{+} a_{\downarrow}=b_{\downarrow}$.

Proof. By induction on the derivation, like for Prop. 2.13. We detail only the rules involving Kleene star:

- (SP): if $a_{\downarrow}=0$ then $\left(1+a \cdot a^{\star}\right)_{\downarrow}=\left(a^{\star}\right)_{\downarrow}=1$ so that we can apply (O); otherwise, $\left(1+a \cdot a^{\star}\right)_{\downarrow}=1+a_{\downarrow} \cdot a_{\downarrow}^{\star}$ and $\left(a^{\star}\right)_{\downarrow}=a_{\downarrow}^{\star}$ : we can apply (SP).

- (SL): suppose that $\vdash a^{\star} \cdot b \leq b$ was obtained using this rule, we have to show that $\vdash^{+}\left(a^{\star} \cdot b\right)_{\downarrow} \leq b_{\downarrow}$. If $b_{\downarrow}=0$ then $\left(a^{\star} \cdot b\right)_{\downarrow}=0$ and we use rule (z). Otherwise $b$ is strict, and either $a_{\downarrow}=0$, in which case $\left(a^{\star} \cdot b\right)_{\downarrow}=1 \cdot b_{\downarrow}$, and we can use rules (OD) and (PI) to get $\vdash^{+} 1 \cdot b_{\downarrow} \leq b_{\downarrow}$; or $a$ is also strict. In the latter case, we use the induction hypothesis: $\vdash^{+}(a \cdot b)_{\downarrow} \leq b_{\downarrow}$, i.e., $\vdash^{+} a_{\downarrow} \cdot b_{\downarrow} \leq b_{\downarrow}$, and we conclude using rule $\left(\mathrm{SL}^{+}\right)$.

- (SR): symmetric to the previous case.

(Note that we implicitly use the fact that normalisation commutes with sum, so that we have $\vdash^{+} a_{\downarrow} \leq b_{\downarrow}$ iff $\vdash^{+}(a+b)_{\downarrow}=b_{\downarrow}$.)

Theorem 2.22. In Kleene algebras, for all $a, b, n, m$ such that $\vdash a, b: n \rightarrow m$, we have $\vdash a=b$ iff $\vdash a=b: n \rightarrow m$.

\subsection{Non-commutative rings.}

Before moving to residuated structures, we briefly discuss the case of non-commutative rings. Indeed, although rings are quite similar to semirings, they cannot be handled in the same way.

Definition 2.23. We define typed rings by the signature $\left\{\cdot_{2},+_{2},-_{1}, 1_{0}, 0_{0}\right\}$, together with the following rules, in addition that from Defs. 2.1 and 2.6, and $\$ 1$.

$$
\frac{\vdash a: n \rightarrow m}{\vdash-a: n \rightarrow m} \text { TI } \quad \frac{\vdash a=b: n \rightarrow m}{\vdash-a=-b: n \rightarrow m} \text { I } \quad \frac{\vdash a: n \rightarrow m}{\vdash a+(-a)=0: n \rightarrow m} \text { PI }
$$


Due to the axiom (PI), we cannot define a simple function to remove annihilators and obtain a factorisation system. Indeed, we have $\vdash a=b$ iff $\vdash a+(-b)=0$, so that strictness amounts to provability; we no longer have a simple syntactical criterion. However, unlike terms of Kleene algebras, terms of non-commutative rings can easily be put in normal form (by expanding the underlying polynomials and ordering monomials lexicographicallyassuming that the set of variables is ordered). This allows us to obtain the untyping theorem by reasoning about the normalisation function.

Let $\langle a\rangle$ denote the normal form of the term $a$ (we do not define formally this standard function here since we are mainly interested in the methodology).

Proposition 2.24. For all $a, b, n, m$, we have

(i) $\vdash a=b$ iff $\langle a\rangle=\langle b\rangle$;

(ii) if $\vdash a: n \rightarrow m$, then $\vdash\langle a\rangle: n \rightarrow m$;

(iii) if $\vdash a: n \rightarrow m$, then $\vdash\langle a\rangle=a: n \rightarrow m$.

Proof.

(i) Standard: this is the correctness and completeness of the untyped decision procedure: two expressions are equal if and only if they share the same normal form.

(ii) By a straightforward induction on the typing derivation.

(iii) Also by induction on the typing derivation, it amounts to replaying the standard correctness proof and checking that it is actually well-typed.

The untyping theorem follows immediately:

Corollary 2.25. In non-commutative rings, for all $a, b, n, m$ such that $\vdash a, b: n \rightarrow m$, we have $\vdash a=b$ iff $\vdash a=b: n \rightarrow m$.

Proof. If $\vdash a=b$ then $\langle a\rangle=\langle b\rangle$ by the point $(i)$ above, which entails $\vdash\langle a\rangle=\langle b\rangle: n \rightarrow m$ by reflexivity since $\vdash\langle a\rangle: n \rightarrow m$ by (ii), from which we deduce $\vdash a=b: n \rightarrow m$ by (iii). The converse implication is straightforward, as in the previous sections.

\section{Residuated LATTiCES}

We now move to our second example, residuated lattices. These structures also admit binary relations as models; they are of special interest to reason algebraically about well-founded relations. For example, residuation is used to prove Newman's Lemma in relation algebras [9]. We start with a simpler structure.

A residuated monoid is a tuple $(X, \leq, \cdot, 1, \backslash, /)$, such that $(X, \leq)$ is a partial order, $(X, \cdot, 1)$ is a monoid whose product is monotonic $\left(a \leq a^{\prime}\right.$ and $b \leq b^{\prime}$ entail $\left.a \cdot b \leq a^{\prime} \cdot b^{\prime}\right)$, and $\backslash, /$ are binary operations, respectively called left and right divisions, characterised by the following equivalences:

$$
a \cdot b \leq c \quad \Leftrightarrow \quad b \leq a \backslash c \quad \Leftrightarrow \quad a \leq c / b
$$

Such a structure can be typed in a natural way, by using the following rules for left and right divisions:

$$
\frac{\vdash c: n \rightarrow m \quad \vdash a: n \rightarrow p}{\vdash a \backslash c: p \rightarrow m} \mathrm{TL} \quad \frac{\vdash c: n \rightarrow m \quad \vdash b: p \rightarrow m}{\vdash c / b: n \rightarrow p} \mathrm{TR}
$$




$$
\begin{array}{cl}
\frac{}{x \vdash x} \mathrm{~V} & \frac{l \vdash a}{\epsilon \vdash 1} \text { Io } \quad \frac{l^{\prime} \vdash a^{\prime}}{l ; l^{\prime} \vdash a \cdot a^{\prime}} \text { ID } \quad \frac{l ; b \vdash a}{l \vdash a / b} \text { IR } \frac{b ; l \vdash a}{l \vdash b \backslash a} \text { IL } \\
\frac{l ; l^{\prime} \vdash a}{l ; 1 ; l^{\prime} \vdash a} \text { EO } & \frac{l ; b ; c ; l^{\prime} \vdash a}{l ; b \cdot c ; l^{\prime} \vdash a} \text { ED } \quad \frac{k \vdash b \quad l ; c ; l^{\prime} \vdash a}{l ; c / b ; k ; l^{\prime} \vdash a} \text { ER } \frac{k \vdash b}{l ; k ; b \backslash c ; l^{\prime} \vdash a} \text { EL }
\end{array}
$$

Figure 1. Gentzen proof system for residuated monoids.

Although we can easily define a set of axioms to capture equalities provable in residuated monoids [19], the transitivity rule $(\mathrm{T})$ becomes problematic in this setting (there is no counterpart to Lemma 2.4). Instead, we exploit a characterisation due to Ono and Komori [31, based on a Gentzen proof system for the full Lambek calculus [26]. Indeed, the "cut" rule corresponding to this system, which plays the role of the transitivity rule, can be eliminated. Therefore, this characterisation allows us to avoid the problems we encountered with standard equational proof systems. In some sense, moving to cut-free proofs corresponds to using a factorisation system, like we did in the previous section (Prop. 2.13).

\subsection{Gentzen proof system for residuated monoids.}

Let $l, k, h$ range over lists of terms, let $l ; k$ denote the concatenation of $l$ and $k$, and let $\epsilon$ be the empty list. The Gentzen proof system is presented on Fig. 1, it relates lists of terms to terms. It is quite standard [19]: there is an axiom rule (V), and, for each operator, an introduction and an elimination rule. The axiom rule can be generalised to terms $(i)$, the cut rule is admissible $(i i)$, and the proof system is correct and complete w.r.t. residuated monoids (iii).

Proposition 3.1. (i) For all a, we have $a \vdash a$.

(ii) For all $l, k, k^{\prime}, a, b$ such that $l \vdash a$ and $k ; a ; k^{\prime} \vdash b$, we have $k ; l ; k^{\prime} \vdash b$.

(iii) For all $a, b$, we have $a \vdash b$ iff $a \leq b$ holds in all residuated monoids.

Proof. Point $(i)$ is easy; see [31, 30, 19] for cut admissibility and completeness.

Type decorations can be added to the proof system in a straightforward way (see Fig. 2). However, using this proof system, we were able to prove the untyping theorem only for the unit-free fragment: we needed to assume that terms have at most one type, which is not true in the presence of 1 . This proof was rather involved, so that we did not manage to circumvent this difficulty in a nice and direct way. Instead, as hinted in the introduction, we move to the following more symmetrical setting.

\subsection{Cyclic MLL.}

The sequent proof system for residuated monoids (Fig. 1) actually corresponds to a noncommutative version of intuitionistic multiplicative linear logic (IMLL) [13]: the product (.) is a non-commutative tensor $(\otimes)$, and left and right divisions $(\backslash, /)$ are the corresponding left and right linear implications $(\multimap, \circ)$. Moreover, it happens that this system is just the intuitionistic fragment of cyclic multiplicative linear logic (MLL) [40]. The untyping theorem turned out to be easier to prove in this setting, which we describe below. 


$$
\begin{aligned}
& \frac{\Gamma(x)=(n, m)}{x \vdash x: n \rightarrow m} \mathrm{v} \quad \frac{l ; l^{\prime} \vdash a: n \rightarrow m}{\epsilon \vdash 1: n \rightarrow n} \text { Iо } \quad \frac{}{l ; 1 ; l^{\prime} \vdash a: n \rightarrow m} \text { Eо } \\
& \frac{l \vdash a: n \rightarrow m \quad l^{\prime} \vdash a^{\prime}: m \rightarrow p}{l ; l^{\prime} \vdash a \cdot a^{\prime}: n \rightarrow p} \text { ID } \quad \frac{l ; b ; c ; l^{\prime} \vdash a: n \rightarrow m}{l ; b \cdot c ; l^{\prime} \vdash a: n \rightarrow m} \text { ED } \\
& \frac{\vdash b: p \rightarrow m \quad l ; b \vdash a: n \rightarrow m}{l \vdash a / b: n \rightarrow p} \operatorname{IR} \frac{\vdash l^{\prime}: m \rightarrow q \quad k \vdash b: n \rightarrow m \quad l ; c ; l^{\prime} \vdash a: p \rightarrow q}{l ; c / b ; k ; l^{\prime} \vdash a: p \rightarrow q} \operatorname{ER} \\
& \frac{\vdash b: n \rightarrow p \quad b ; l \vdash a: n \rightarrow m}{l \vdash b \backslash a: p \rightarrow m} \mathrm{IL} \quad \frac{\vdash l: p \rightarrow m \quad k \vdash b: m \rightarrow n \quad l ; c ; l^{\prime} \vdash a: p \rightarrow q}{l ; k ; b \backslash c ; l^{\prime} \vdash a: p \rightarrow q} \mathrm{EL}
\end{aligned}
$$

Figure 2. Typed Gentzen proof system for residuated monoids.

We assume a copy $\mathcal{X}^{\perp}$ of the set of variables $(\mathcal{X})$, and we denote by $x^{\perp}$ the corresponding elements which we call dual variables. From now on, we shall consider terms with both kinds of variables: $T\left(\Sigma+X+X^{\perp}\right)$. We keep an algebraic terminology to remain consistent with the previous sections; notice that using terminology from logic, a term is a formula and a variable is an atomic formula.

Definition 3.2. Typed $M L L$ terms are defined by the signature $\left\{\otimes_{2}, \gamma_{2}, 1_{0}, \perp_{0}\right\}$, together with the following typing rules:

$$
\begin{aligned}
& \frac{\Gamma(x)=(n, m)}{\vdash x: n \rightarrow m} \mathrm{TV} \quad \frac{\vdash a: n \rightarrow m \quad \vdash b: m \rightarrow p}{\vdash 1: n \rightarrow n} \mathrm{~T}_{1} \quad \frac{\vdash a \otimes b: n \rightarrow p}{\vdash} \mathrm{T}_{\otimes} \\
& \frac{\Gamma(x)=(n, m)}{\vdash x^{\perp}: m \rightarrow n} \mathrm{TV}^{\perp} \quad \frac{\vdash a: n \rightarrow m \quad \vdash b: m \rightarrow p}{\vdash \perp: n \rightarrow n} \mathrm{~T}_{\perp} \quad \frac{\vdash a \not 8 b: n \rightarrow p}{\vdash a}
\end{aligned}
$$

Tensor $(\otimes)$ and par $(28)$ are typed like the previous dot operation; bottom $(\perp)$ is typed like the unit (1); dual variables are typed by mirroring the types of the corresponding variables. We extend type judgements to lists of terms as follows:

$$
\frac{\vdash a: n \rightarrow m \quad \vdash l: m \rightarrow p}{\vdash \epsilon: n \rightarrow n} \mathrm{TE} \text { TC }
$$

(be careful not to confuse $\vdash a, b: n \rightarrow m$, which indicates that both $a$ and $b$ have type $n \rightarrow m$, with $\vdash a ; b: n \rightarrow m$, which indicates that the list $a ; b$ has type $n \rightarrow m)$. Linear negation is defined over terms and lists of terms as follows:

$$
\begin{array}{cccc}
(x)^{\perp} \triangleq x^{\perp} & 1^{\perp} \triangleq \perp & (a \otimes b)^{\perp} \triangleq b^{\perp} \gamma a^{\perp} & (a ; l)^{\perp} \triangleq l^{\perp} ; a^{\perp} \\
\left(x^{\perp}\right)^{\perp} \triangleq x & \perp^{\perp} \triangleq 1 & (a \ngtr b)^{\perp} \triangleq b^{\perp} \otimes a^{\perp} & \epsilon^{\perp} \triangleq \epsilon
\end{array}
$$

Note that since we are in a non-commutative setting, negation has to reverse the arguments of tensors and pars, as well as lists. Negation is involutive and mirrors type judgements:

Lemma 3.3. For all $l, l^{\perp \perp}=l$; for all $l, n, m, \vdash l: n \rightarrow m$ iff $\vdash l^{\perp}: m \rightarrow n$. 


$$
\begin{aligned}
& { }_{\vdash 1: n} 1 \quad \frac{\vdash l: n}{\vdash \perp ; l: n} \perp \quad \frac{\vdash l ; a: n \quad \vdash b ; k: n}{\vdash l ; a \otimes b ; k: n} \otimes \quad \frac{\vdash a ; b ; l: n}{\vdash a \text { Pb;l:n}} \text { ช } \\
& \frac{\Gamma(x)=(n, m)}{\vdash x^{\perp} ; x: m} \mathrm{~A} \quad \frac{\vdash a: n \rightarrow m \quad \vdash l ; a: m}{\vdash a ; l: n} \mathrm{E}
\end{aligned}
$$

Figure 3. Typed proof system for Cyclic MLL.

If we were using a two-sided presentation of MLL, judgements would be of the form $l \vdash k$ : $m \rightarrow n$, intuitively meaning " $l \vdash k$ is derivable in cyclic MLL, and lists $l$ and $k$ have type $m \rightarrow n$ ". Instead, we work with one-sided sequents to benefit from the symmetrical nature of MLL. At the untyped level, this means that we replace $l \vdash k$ with $\vdash l^{\perp} ; k$. According to the previous intuitions, the list $l^{\perp} ; k$ has a square type $n \rightarrow n$ : the object $m$ is hidden in the concatenation, so that it suffices to record the outer object $(n)$. Judgements finally take the form $\vdash l: n$, meaning "the one-sided MLL sequent $\vdash l$ is derivable at type $n \rightarrow n$ ".

Definition 3.4. Typed cyclic $M L L$ is defined by the sequent calculus from Fig. 3.

Except for type decorations, the system is standard: the five first rules are the logical rules of MLL [13]. Rule (E) is the only structural rule, this is a restricted form of the exchange rule, yielding cyclic permutations: sequents have to be thought of as rings [40]. As before, we added type decorations in a minimal way, so as to ensure that derivable sequents have square types, as explained above:

Lemma 3.5. For all $l, n$, if $\vdash l: n$ then $\vdash l: n \rightarrow n$.

We now give a graphical interpretation of the untyping theorem, using proof nets. Since provability is preserved by cyclic permutations, one can draw proof structures by putting the terms of a sequent on a circle [40]. For example, a proof $\pi$ of a sequent $\vdash l_{0}, \ldots, l_{i}$ will be represented by a proof net whose interface is given by the left drawing below.
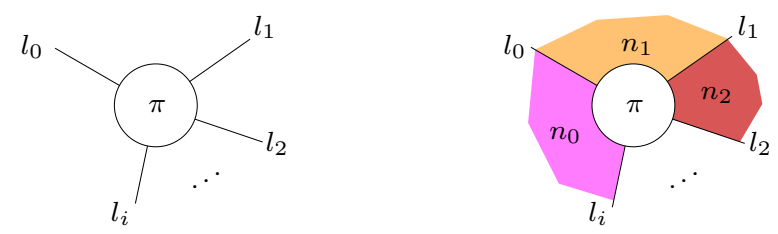

Suppose now that the corresponding list admits a square type: $\vdash l: n \rightarrow n$, i.e., $\forall j \leq$ $i, \vdash l_{j}: n_{j} \rightarrow n_{j+1}$, for some $n_{0}, \ldots, n_{i+1}$ with $n=n_{0}=n_{i+1}$. One can add these type decorations as background colours, in the areas delimited by terms, as we did on the right-hand side.

The logical rules of the proof system (Fig. 3) can then be represented by the proof net constructions from Fig. 4 (thanks to this sequent representation, the exchange rule (E) is implicit). Since these constructions preserve planarity, all proof nets are planar [3], and the idea of background colours makes sense. Moreover, they can be coloured in a consistent way, so that typed derivations correspond to proof nets that can be entirely and consistently coloured. Therefore, one way to prove the untyping theorem consists in showing that any proof net whose outer interface can be coloured can be coloured entirely. As an example, we 

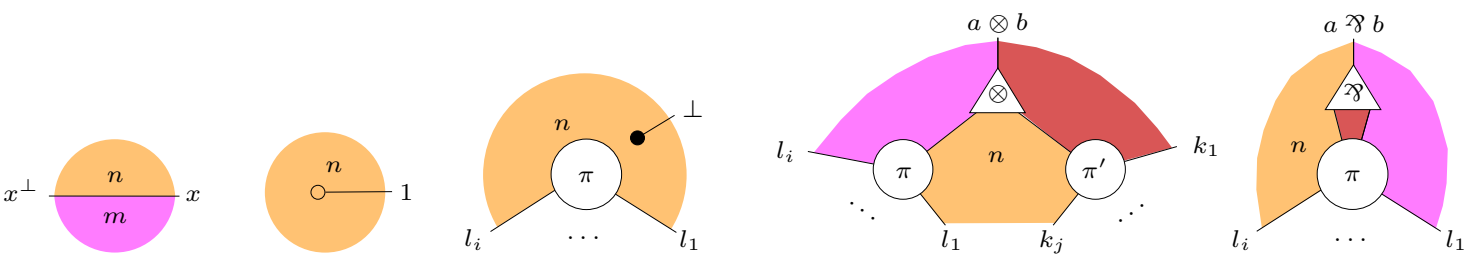

Figure 4. Proof nets for Cyclic MLL.

give an untyped derivation below, together with the corresponding proof net. Assuming that $\Gamma(x)=n \rightarrow m$ and $\Gamma(y)=m \rightarrow p$, the conclusion has type $p \rightarrow p$, and the outer interface of the proof net can be coloured (here, with colours $p$ and $n$ ). The untyping theorem will ensure that there exists a typed proof; indeed, the whole proof net can be coloured in a consistent way.

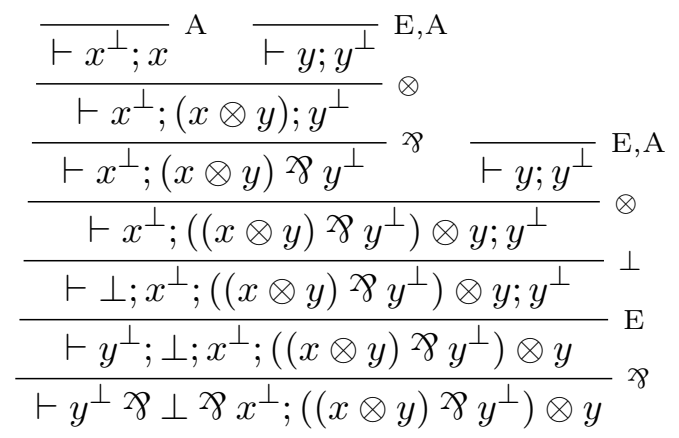

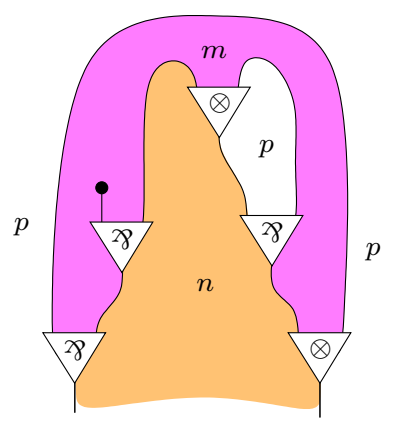

We now embark in the proof of the untyping theorem for cyclic MLL; the key property is that the types of derivable sequents are all squares:

Proposition 3.6. If $\vdash l$ and $\vdash l: n \rightarrow m$, then $n=m$.

Proof. We proceed by induction on the untyped derivation $\vdash l$, but we prove a stronger property: "the potential types of all cyclic permutations of $l$ are squares", i.e., for all $h, k$ such that $l=h ; k$, for all $n, m$ such that $\vdash k ; h: n \rightarrow m, n=m$. The most involved case is that of the tensor rule. Using symmetry arguments, we can assume that the cutting point belongs to the left premise: the conclusion of the tensor rule is $\vdash l ; l^{\prime} ; a \otimes b ; k$, we suppose that the induction hypothesis holds for $l ; l^{\prime} ; a$ and $b ; k$, and knowing that $\vdash l^{\prime} ; a \otimes b ; k ; l: n \rightarrow m$, we have to show $n=m$. Clearly, we have $\vdash l^{\prime} ; a: n \rightarrow p, \vdash b ; k: p \rightarrow q$, and $\vdash l: q \rightarrow m$ for some $p, q$. By induction on the second premise, we have $p=q$, so that $\vdash l^{\prime} ; a ; l: n \rightarrow m$. Since the latter list is a cyclic permutation of $l ; l^{\prime} ; a$, we can conclude with the induction hypothesis on the first premise.

Theorem 3.7. In cyclic $M L L$, if $\vdash l: n \rightarrow n$, then we have $\vdash l$ iff $\vdash l: n$.

Proof. The right-to-left implication is straightforward; for the direct implication, we proceed by induction on the untyped derivation. The previous proposition is required in the case of the tensor rule: we know that $\vdash l ; a, \vdash b ; k$, and $\vdash l ; a \otimes b ; k: n \rightarrow n$, and we have to show that $\vdash l ; a \otimes b ; k: n$. Necessarily, there is some $m$ such that $\vdash l ; a: n \rightarrow m$ and $\vdash b ; k: m \rightarrow n$; moreover, by Prop. 3.6, $n=m$. Therefore, we can apply the induction hypotheses (so that $\vdash l ; a: n$ and $\vdash b ; k: n$ ) and we conclude with the typed tensor rule. 


\subsection{Intuitionistic fragment.}

To deduce that the untyping theorem holds in residuated monoids, it suffices to show that the typed version of the proof system from 3.1 corresponds to the intuitionistic fragment of the proof system from Fig. 3. This is well-known for the untyped case, and type decorations do not add particular difficulties. Therefore, we just give a brief overview of the extended proof.

The idea is to define the following families of input and output terms (Danos-Regnier polarities [37, 4]), and to work with sequents composed of exactly one output term and an arbitrary number of input terms.

$$
\begin{aligned}
& i::=x^{\perp}|\perp| i \not \gamma i|i \otimes o| o \otimes i \\
& o::=x|1| o \otimes o \mid i \text { ช o }
\end{aligned}
$$

Negation $\left(-^{\perp}\right)$ establishes a bijection between input and output terms. Terms of residuated monoids (IMLL formulae) are encoded into output terms as follows.

$$
\begin{array}{lll}
\lfloor a \cdot b\rfloor \triangleq\lfloor a\rfloor \otimes\lfloor b\rfloor & \lfloor a / b\rfloor \triangleq\lfloor a\rfloor \not \supset\lfloor b\rfloor^{\perp} & \lfloor x\rfloor \triangleq x \\
\lfloor 1\rfloor \triangleq 1 & \lfloor a \backslash b\rfloor \triangleq\lfloor a\rfloor^{\perp}>\lfloor b\rfloor &
\end{array}
$$

This encoding is a bijection between IMLL terms and MLL output terms; it preserves typing judgements:

Lemma 3.8. For all $a, n, m$, we have $\vdash a: n \rightarrow m$ iff $\vdash\lfloor a\rfloor: n \rightarrow m$.

(Note that we heavily rely on overloading to keep notation simple.) The next proposition shows that we actually obtained a fragment of typed cyclic MLL; it requires the lemma below: input-only lists are not derivable. The untyping theorem for residuated monoids follows using Thm. 3.7 .

Lemma 3.9. If $\vdash l$, then $l$ contains at least one output term.

Proposition 3.10. If $\vdash l, a: n \rightarrow m$, then $l \vdash a: n \rightarrow m$ iff $\vdash\lfloor l\rfloor^{\perp} ;\lfloor a\rfloor: m$.

Proof. The forward implication is proved by an induction on the sequent derivation. For the reverse direction, we actually prove the following stronger property, by induction on the untyped MLL derivation:

"for all $h, a, k, n, m$ such that we have $\vdash\lfloor h\rfloor^{\perp} ;\lfloor a\rfloor ;\lfloor k\rfloor^{\perp}, \vdash h ; k: n \rightarrow m$, and $\vdash a: n \rightarrow m$, we have $h ; k \vdash a: n \rightarrow m$ ".

This generalisation is required to handle the exchange rule. We detail only the key cases:

- If the tensor rule was used last, on the output term (which was thus of the form $\lfloor a \cdot b\rfloor=$ $\lfloor a\rfloor \otimes\lfloor b\rfloor):$

$$
\frac{\vdash\lfloor h\rfloor^{\perp} ;\lfloor a\rfloor \quad \vdash\lfloor b\rfloor ;\lfloor k\rfloor^{\perp}}{\vdash\lfloor h\rfloor^{\perp} ;\lfloor a\rfloor \otimes\lfloor b\rfloor ;\lfloor k\rfloor^{\perp}} \otimes
$$

Since $\vdash a \cdot b: n \rightarrow m$, and $\vdash h ; k: n \rightarrow m$, we have $p, q$ such that $\vdash a: n \rightarrow p$, $\vdash b: p \rightarrow m, \vdash h: n \rightarrow q$ and $\vdash k: q \rightarrow m$. Therefore, by Lemmas 3.3 and 3.8, we have $\vdash\lfloor h\rfloor^{\perp} ;\lfloor a\rfloor: q \rightarrow p$, whence $p=q$ by Prop. 3.6. We can thus apply the induction hypothesis to the two premises to obtain $h \vdash a: n \rightarrow p$ and $k \vdash b: p \rightarrow m$ (using an empty sequence in both cases). We conclude using rule (ID) from Fig. 1 . 
- If the tensor rule was used last, on one of the input terms, say on $b$ in $h=h_{1} ; b ; h_{2}$, with $\lfloor b\rfloor^{\perp}=c \otimes d:$

$$
\frac{\vdash\left\lfloor h_{2}\right\rfloor^{\perp} ; c \quad \vdash d ;\left\lfloor h_{1}\right\rfloor^{\perp},\lfloor a\rfloor, k^{\perp}}{\vdash\left\lfloor h_{2}\right\rfloor^{\perp} ; c \otimes d ;\left\lfloor h_{1}\right\rfloor^{\perp} ;\lfloor a\rfloor ;\lfloor k\rfloor^{\perp}} \otimes
$$

Since $\lfloor h\rfloor_{2}^{\perp} ; c$ is provable and $\lfloor h\rfloor_{2}^{\perp}$ contains only input terms, $c$ is necessarily an output term by Lemma 3.9. Therefore there is only one possibility ensuring $\lfloor b\rfloor=d^{\perp} \not 8 c^{\perp}$ : the term $b$ must be of the form $d^{\prime} / c^{\prime}$, with $\left\lfloor d^{\prime}\right\rfloor=d^{\perp}$ and $\left\lfloor c^{\prime}\right\rfloor=c$.

We have $\vdash h_{1} ; d^{\prime} / c^{\prime} ; h_{2} ; k: n \rightarrow m$ and $\vdash a: n \rightarrow m$, i.e., $\vdash h_{1}: n \rightarrow p, \vdash d^{\prime}: p \rightarrow q$, $\vdash c^{\prime}: r \rightarrow q, \vdash h_{2}: r \rightarrow s$, and $\vdash k: s \rightarrow m$ for some $p, q, r, s$. We first notice that the provable sequent $\left\lfloor h_{2}\right\rfloor^{\perp} ; c$ has type $s \rightarrow q$, so that $s=q$ by Prop. 3.6. By induction, we then deduce $h_{2} \vdash c^{\prime}: r \rightarrow q$ and $h_{1} ; d^{\prime} ; k \vdash a: n \rightarrow m$, and we conclude using rule (ER) from Fig. 1.

Corollary 3.11. In residuated monoids, if $\vdash l, a: n \rightarrow m$, then we have $l \vdash a$ iff $l \vdash a$ : $n \rightarrow m$.

\subsection{Residuated lattices: additives.}

The Gentzen proof system we presented for residuated monoids (Fig. 1) was actually designed for residuated lattices [31], obtained by further requiring the partial order $(X, \leq)$ to be a lattice $(X, \vee, \wedge)$. Binary relations fall into this family, by considering set-theoretic unions and intersections. The previous proofs scale without major difficulty: on the logical side, this amounts to considering the additive binary connectives $(\oplus, \&)$. By working in multiplicative additive linear logic (MALL) without additive constants, we get an untyping theorem for involutive residuated lattices [39]; we deduce the untyping theorem for residuated lattices by considering the corresponding intuitionistic fragment (see [33] for proofs).

On the contrary, and rather surprisingly, the theorem breaks if we include additive constants $(0, \top)$, or equivalently, if we consider bounded residuated lattices. The corresponding typing rules are given below, together with the logical rule for top (there is no rule for zero).

$$
\overline{\vdash 0: n \rightarrow m}^{\mathrm{T}_{0}} \quad \frac{\vdash l: m \rightarrow n}{\vdash \top: n \rightarrow m} \mathrm{~T}_{\top} \quad \frac{\vdash}{\vdash \top l: n}
$$

The sequent $x^{\perp} \otimes \top ; y^{\perp} ; \top \otimes x$ gives a counter-example. This sequent basically admits the two following untyped proofs:

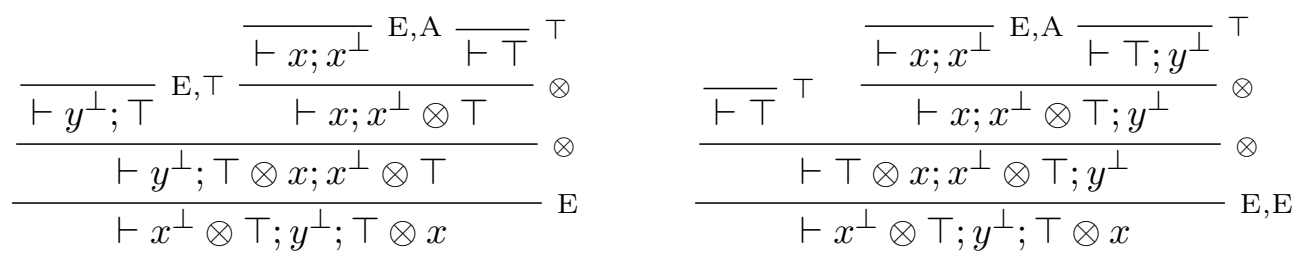

However, this sequent admits the square type $m \rightarrow m$ whenever $\Gamma(x)=(n, m)$ and $\Gamma(y)=(p, q)$, while the above proofs cannot be typed unless $n=q$ or $n=p$, respectively. Graphically, these proofs correspond to the proof nets below (where the proof net construction for rule $(T)$ is depicted on the left-hand side); these proof nets cannot be coloured unless $n=q$ or $n=p$. 

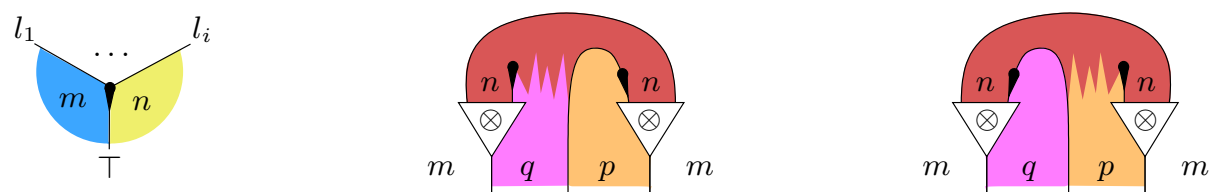

This counter-example for MALL also gives a counter-example for IMALL: the above proofs translate to intuitionistic proofs of $y \cdot(T \backslash x) \vdash \top \cdot x$, which is also not derivable in the typed setting, unless $n=q$ or $n=p$.

The problem is actually even stronger: while $S \cdot(\top \backslash R) \subseteq \top \cdot R$ holds for all homogeneous binary relations $R, S$ (by the above untyped proofs, for example), this law does not hold for arbitrary heterogeneous relations (see Remark 3.12 below). This shows that we cannot always reduce the analysis of typed structures to that of the underlying untyped structures. Here, the equational theory of heterogeneous binary relations does not reduce to the equational theory of homogeneous binary relations.

Remark 3.12. The containment $S \cdot(T \backslash R) \subseteq \top \cdot R$ does not necessarily hold for all heterogeneous binary relations $R, S$, although it holds for all heterogeneous binary relations on non-empty sets.

Proof. Let $A, B, C, D$ be four sets, let $R \subseteq B \times C$ be a binary relation from $B$ to $C$, and let $S \subseteq D \times A$ be a binary relation from $D$ to $A$. To be precise, we denote by $\top_{X, Y}$ the full relation between sets $X$ and $Y(X \times Y)$, and the containment from the statement can be rewritten as

$$
S \cdot\left(\top_{B, A} \backslash R\right) \subseteq \top_{D, B} \cdot R
$$

For all relations $T \subseteq B \times A$, the relation $T \backslash R$ is characterised as follows:

$$
T \backslash R=\{(i, j) \in A \times C \mid \forall k \in B,(k, i) \in T \rightarrow(k, j) \in R\} .
$$

- if $B$ is the empty set, then $R=\top_{D, B} \cdot R=\emptyset$, and by the above characterisation, we have $\top_{B, A} \backslash R=A \times C$. Therefore, we can contradict the containment by taking any non-empty relation for $S$. (Note that this cannot happen in an homogeneous setting: we have $A=B=C=D$ so that taking the empty set for $B$ forces both $R$ and $S$ to be empty.)

- if $B$ is not empty, then we have

$$
\begin{aligned}
\top_{B, A} \backslash R & =\left\{(i, j) \in A \times C \mid \forall k \in B,(k, i) \in \top_{B, A} \rightarrow(k, j) \in R\right\} \\
& =\{(i, j) \in A \times C \mid \forall k \in B,(k, j) \in R\} \\
& \subseteq\{(i, j) \in A \times C \mid \exists k \in B,(k, j) \in R\} \\
& =\left\{(i, j) \in A \times C \mid \exists k \in B,(i, k) \in \top_{A, B} \wedge(k, j) \in R\right\} \\
& =\top_{A, B} \cdot R ;
\end{aligned}
$$

Therefore, since $S \subseteq \top_{D, A}$, we can conclude:

$$
S \cdot\left(\top_{B, A} \backslash R\right) \subseteq \top_{D, A} \cdot \top_{A, B} \cdot R \subseteq \top_{D, B} \cdot R .
$$



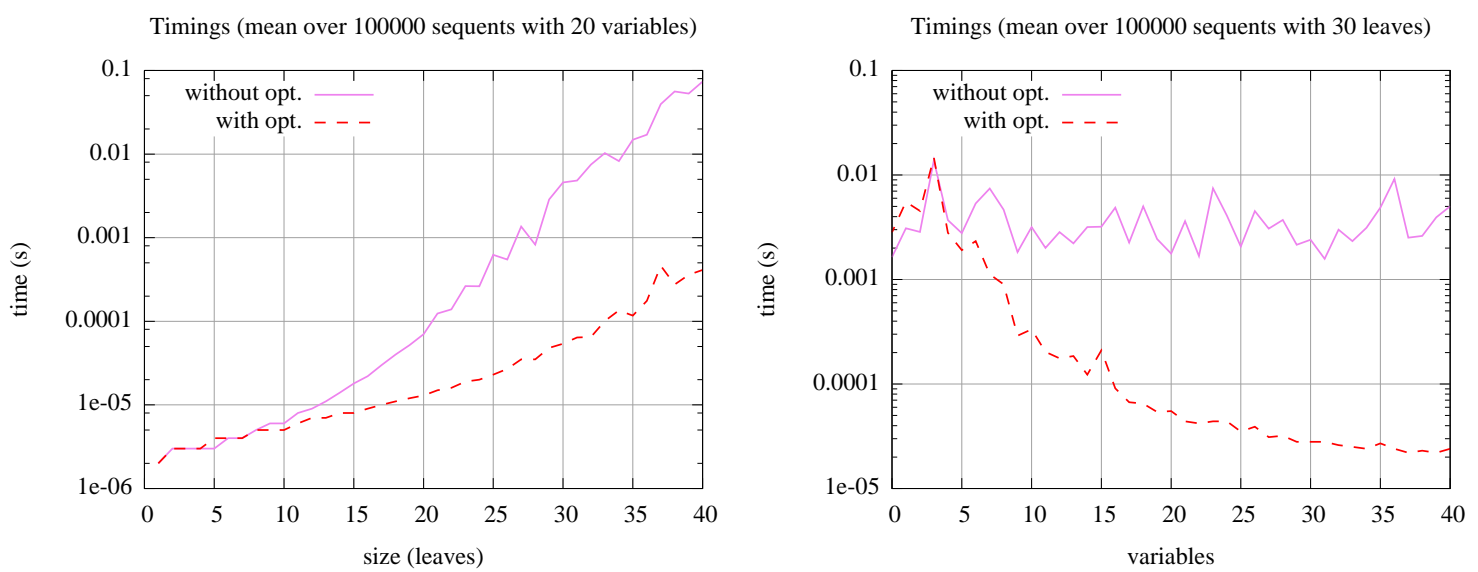

Figure 5. Searching times for focused proof search with and without optimisation.

We do not know whether relations on empty sets are required to get such a counter-example in the model of binary relations. In other words, for the signature of bounded residuated lattices, does the equational theory of heterogeneous binary relations on non-empty sets reduce to the equational theory of homogeneous binary relations?

\section{IMPROVING PROOF SEARCH FOR RESIDUATED STRUCTURES.}

The sequent proof systems we mentioned in the previous section have the sub-formula property, so that provability is decidable in each case, using a simple proof search algorithm [30]. Surprisingly, the concept of type can be used to cut off useless branches. Indeed, recall Prop. 3.6. "the types of any derivable sequent are squares". By contrapositive, given an untyped sequent $l$, one can easily compute an abstract 'most general type and environment' $(n \rightarrow m, \Gamma)$, such that $\Gamma \vdash l: n \rightarrow m$ holds (taking $\mathbb{N}$ as the set of objects, for example); if $n \neq m$, then the sequent is not derivable, and proof search can fail immediately on this sequent.

We did some experiments with a simple prototype [33]: we implemented focused [2] proof search for cyclic MALL, i.e., a recursive algorithm composed of an asynchronous phase which is deterministic and a synchronous phase, where branching occurs (e.g., when applying the tensor rule $(\otimes))$. The optimisation consists in checking that the most general type of the sequent is square before entering the synchronous phases. The overall complexity remains exponential (provability is NP-complete [32] - PSPACE-complete with additives [20]) but we get an exponential speed-up: we can abort proof search immediately on approximately two sequents out of three.

The experimental results are given on Fig. 5 and 6-raw data is available from [33]. We generate (pseudo) random sequents in normal form with respect to the laws of neutral elements for multiplicative constants $(1$ and $\perp$ ), with a given number of leaves (variables, dual variables or constants), and where variables are picked in a set of the specified size. E.g., $a \otimes \perp ; b^{\perp}$ is a sequent with three leaves and two variables, which can also be considered as a sequent with three leaves and four variables, where two variables are not used. 


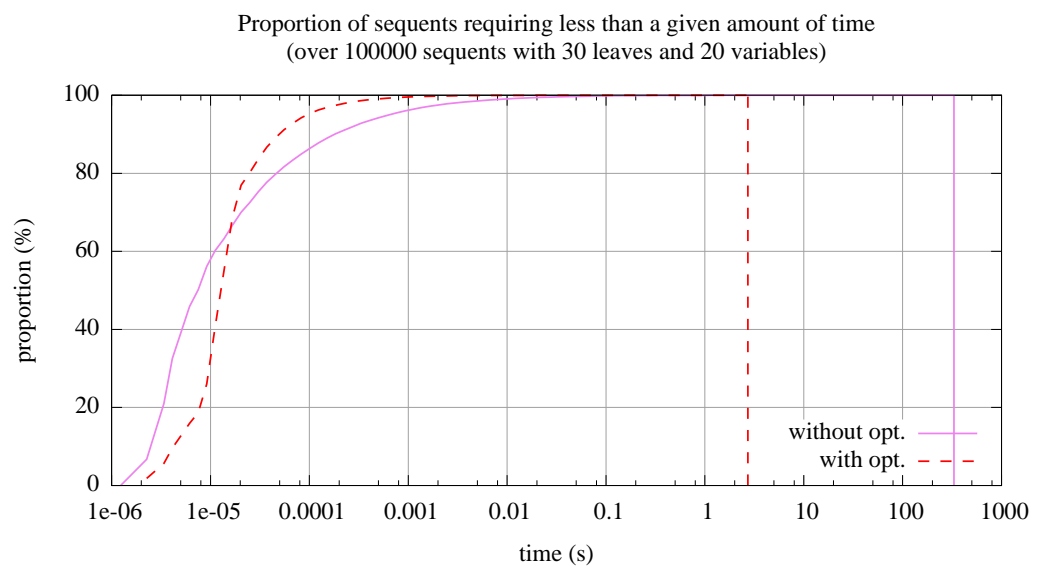

Figure 6. Distribution of searching times.

Each point of Fig. 5 was obtained by timing focused proof search with and without optimisation, on a set of 100000 sequents with the given characteristics: fixed number of variables and varying size on the left-hand side, fixed size and varying number of variables on the right-hand side. While the optimisation introduces a small amount of overhead for very small sequents or sequents with few variables, we gain more than one order of magnitude for larger sequents. One can also notice that the more variables are available, the more efficient the optimisation is: indeed, sequents with a lot of different variables tend to have non-square types more easily, so that they can be ruled out more frequently.

We did not report standard deviation in Fig. 5 since it does not make sense in this setting: we have an unbounded set of potential values, and the actual complexity of proof search is highly stochastic. Instead, we computed the distribution of searching times: Fig. 6 shows the proportion of sequents that are solved in a given amount of time, among sequents with a fixed size and number of variables-here, 30 leaves and 20 variables. While $60 \%$ of the sequents are solved in less that $10^{-5} \mathrm{~s}$ (with or without optimisation), some of them require much more time: up to five minutes without optimisation, and up to three seconds with the optimisation. All in all, the overhead which is paid on 'easily solved' sequents gets compensated by the drastic improvement on 'harder' sequents.

\section{CONClusions AND Directions FOR FUture WORK}

We proved untyping theorems for several standard structures, allowing us to extend decidability results to the typed settings, and to discover an optimisation of proof search for cyclic linear logic. All results have been formally checked [33] with the Coq proof assistant.

The untyping theorem for typed Kleene algebras is quite important in the ATBR Coq library [5]: it allows one to use our tactic for Kleene algebras in typed settings, and, in particular, with heterogeneous binary relations. The underlying decision procedure being quite involved, we can hardly imagine proving its soundness with respect to typed settings in a direct way. Even writing a type-preserving version of the algorithm seems challenging.

At another level, we used the untyping theorem for semirings in order to formalise Kozen's completeness proof [22] for Kleene algebras. Indeed, this proof heavily relies on 
matrix constructions, so that having adequate lemmas and tactics for working with possibly rectangular matrices was a big plus: this allowed us to avoid the ad-hoc constructions Kozen used to inject rectangular matrices into square ones.

\subsection{References and related work.}

The relationship between residuated lattices and substructural logics is due to Ono and Komori [31]; see [11] for a thorough introduction. Cyclic linear logic was suggested by Girard and studied by Yetter [40]. To the best of our knowledge, the idea of adding types to the above structures is new. The axiomatisation of Kleene algebras is due to Kozen [22].

Our typed structures can be seen as very special cases of partial algebras [6], where the domain of partial operations is defined by typing judgements. Similarly, one could use many-sorted algebras [16] to mimic types using sorts. Several encodings from partial algebras to total ones were proposed in the literature [28, 7]. Although they are quite general, these results do not apply here: these encodings do not preserve the considered theory since they need to introduce new symbols and equations; as a consequence, ordinary untyped decision procedures can no longer be used after the translation. Dojer has shown that under some conditions, convergent term rewriting systems for total algebras can be used to prove existence equations in partial algebras [8]. While it seems applicable to semirings, this approach does not scale to Kleene algebras or residuated lattices, for which decidability does not arise from a term rewriting system.

The idea of proving typed equations from untyped ones also appears in the context of "Pure Type Systems" (PTSs), where one can use either an untyped conversion rule, or a typed equality judgement. Whether these two possible presentations were equivalent was open for some time [12]; Adams has shown that this is the case for "functional" PTSs [1], Herbelin and Siles recently generalised the result to all PTSs [38]. Although the types we use here are quite basic (i.e., a type is just a pair of abstract objects), our use of cut-free proof systems and factorisation systems is reminiscent to their use of the Church-Rosser property. Note however that unlike in functional programming languages, where one usually relies on a Hindley-Milner type inference algorithm [17, 27] to rule out ill-typed programs, no inference algorithm is required with the algebraic theories presented here: such an algorithm would always succeed since an untyped proof systematically yields a typed proof.

Closer to our work is that of Kozen, who first proposed the idea of untyping typed Kleene algebras, in order to avoid the aforementioned matrix constructions [24]. He provided a different answer, however: using model-theoretic arguments, he proved an untyping theorem for the universal theory of "1-free Kleene algebras". The restriction to 1-free expressions is required, as shown by the following counter-example: $\vdash 0=1 \Rightarrow a=b$ is a theorem of semirings, although there are non trivial typed semirings where $0=1$ holds at some types (e.g., empty matrices), while $a=b$ is not universally true at other types.

\subsection{Handling other structures.}

Action algebras [35, 18] are a natural extension of the structures we studied in this paper: they combine the ingredients from residuated lattices and Kleene algebras. In this setting, left and right divisions make it possible to obtain a variety rather than a quasivariety: inference rules (SL) and (SR), about the star operation, can be replaced by the following equational axioms:

$$
\overline{\vdash(a \backslash a)^{\star}=a \backslash a} \mathrm{SL}^{\prime} \quad \overline{\vdash(a / a)^{\star}=a / a} \mathrm{SR}^{\prime}
$$


Although we do not know whether the untyping theorem holds in this case, we can think of two strategies to tackle this problem: 1) find a cut-free extension of the Gentzen proof system for residuated lattices and adapt our current proof - such an extension is left as an open question in [18, it would possibly entail decidability of the equational theory of action algebras; 2) find a "direct" proof of the untyping theorem for residuated monoids, without using a Gentzen proof system, so that the methodology we used for Kleene algebras can be extended. Also note that we necessarily have to exclude the annihilator element (0): with divisions, top $(T)$ can be defined as $0 / 0$, so that the counter-example for bounded residuated lattices (\$3.4) applies. Consistently, there is no way to remove this element using a factorisation system: expressions like $a \cdot \top$ cannot be simplified.

Kleene algebras with tests [23] are another extension of Kleene algebras, which is useful in program verification. Their equational theory is decidable, but one cannot rely on a factorisation system to remove annihilators in this setting: like for rings $(\$ 2.4)$, the complement operation of the Boolean algebra is problematic. Moreover, like for Kleene algebras, there are no known notions of normal form in Kleene algebras with tests, so that the approach we described in 2.4 is not possible. Nonetheless, the untyping theorem is likely to hold for these structures since the Boolean algebras of tests are inherently homogeneous.

Finally, although our methodology for semirings can be adapted to handle the case of allegories [10] (see [33] for a proof), the cases of distributive and division allegories - where left and right divisions are added-remains open.

Finally, although our methodology for semirings can be adapted to handle the case of allegories [10] (see [33] for a proof), the cases of distributive allegories as well as division allegories - where left and right divisions are added — remain open.

\subsection{Towards a generic theory.}

The typed structures we focused on can be described in terms of enriched categories, and the untyping theorems can be rephrased as asserting the existence of faithful functors to one-object categories. It would therefore be interesting to find out whether category theory may help to define a reasonable class of structures for which the untyping theorem holds. In particular, how could we exclude the counter-example with additive constants in MALL?

For structures that are varieties, another approach would consist of using term rewriting theory to obtain generic factorisation theorems (Lemma 2.13, which we used to handle the annihilating element in semirings, would become a particular case). This seems rather difficult, however, since these kind of properties are quite sensitive to the whole set of operations and axioms that are considered.

\section{ACKNOWLEDGEMENTS.}

We are grateful to Olivier Laurent and Tom Hirschowitz for the highly stimulating discussions we had on linear logic and about this work.

\section{REFERENCES}

[1] R. Adams. Pure type systems with judgemental equality. J. Funct. Program., 16(2):219-246, 2006.

[2] J.-M. Andreoli. Logic programming with focusing proofs in linear logic. Journal of Logic and Computation, 2(3):297-347, 1992.

[3] G. Bellin and A. Fleury. Planar and braided proof-nets for MLL with mix. Archive for Mathematical Logic, 37:309-325, 1998. 
[4] G. Bellin and P. Scott. On the $\pi$-calculus and linear logic. TCS, 135:11-65, 1994.

[5] T. Braibant and D. Pous. An efficient Coq tactic for deciding Kleene algebras. In Proc. ITP, volume 6172 of $L N C S$, pages 163-178. Springer, 2010.

[6] P. Burmeister. Partial algebra — an introductory survey. In Algebras and Orders, volume 389 of NATO ASI, pages 1-70. Kluwer Pub., 1993.

[7] R. Diaconescu. An encoding of partial algebras as total algebras. Inf. Process. Lett., 109(23-24):1245$1251,2009$.

[8] N. Dojer. Applying term rewriting to partial algebra theory. Fund. Inf., 63(4):375-384, 2004.

[9] H. Doornbos, R. Backhouse, and J. van der Woude. A calculational approach to mathematical induction. TCS, 179(1-2):103-135, 1997.

[10] P. Freyd and A. Scedrov. Categories, Allegories. North Holland, 1990.

[11] N. Galatos, P. Jipsen, T. Kowalski, and H. Ono. Residuated lattices: an algebraic glimpse at substructural logics. Stud. in Log. and Found. of Math., 151:532, 2007.

[12] H. Geuvers and B. Werner. On the Church-Rosser property for expressive type systems and its consequences for their metatheoretic study. In Proc. LICS, pages 320-329. IEEE, 1994.

[13] J.-Y. Girard. Linear logic. TCS, 50:1-102, 1987.

[14] B. Grégoire and A. Mahboubi. Proving equalities in a commutative ring done right in Coq. In Proc. TPHOLs, volume 3603 of LNCS, pages 98-113. Springer, 2005.

[15] J. Harrison. A HOL decision procedure for elementary real algebra. In HUG, volume 780 of $L N C S$, pages 426-435. Springer, 1993.

[16] P. Higgins. Algebras with a scheme of operators. Math. Nach., 27:115-132, 1963.

[17] R. Hindley. The principal type-scheme of an object in combinatory logic. Transactions of the American Mathematical Society, 146:29-60, 1969.

[18] P. Jipsen. From semirings to residuated Kleene lattices. Stud. Log., 76(2):291-303, 2004.

[19] P. Jipsen and C. Tsinakis. A survey of residuated lattices. Ord. Alg. Struct., 2002.

[20] M. Kanovich. The complexity of neutrals in linear logic. In Proc. LICS, pages 486-495. IEEE, 1995.

[21] S. C. Kleene. Representation of events in nerve nets and finite automata. In Automata Studies, pages 3-41. Princeton University Press, 1956.

[22] D. Kozen. A completeness theorem for Kleene algebras and the algebra of regular events. Inf. and Comput., 110(2):366-390, 1994.

[23] D. Kozen. Kleene algebra with tests. Trans. PLS, 19(3):427-443, 1997.

[24] D. Kozen. Typed Kleene algebra. Technical Report 98-1669, Cornell Univ., 1998.

[25] D. Krob. Complete systems of B-rational identities. TCS, 89(2):207-343, 1991.

[26] J. Lambek. The mathematics of sentence structure. American Mathematical Monthly, 65:154-170, 1958.

[27] R. Milner. A theory of type polymorphism in programming. J. Comput. Syst. Sci., 17(3):348-375, 1978.

[28] T. Mossakowski. Relating CASL with other specification languages: the institution level. TCS, 286(2):367-475, 2002.

[29] M. Norrish. Complete integer decision procedures as derived rules in HOL. In Proc. TPHOLs, volume 2758 of $L N C S$, pages 71-86. Springer, 2003.

[30] M. Okada and K. Terui. The finite model property for various fragments of intuitionistic linear logic. J. Sym. Log., 64(2):790-802, 1999.

[31] H. Ono and Y. Komori. Logics without the contraction rule. J. Sym. Log., 50(1):169-201, 1985.

[32] M. Pentus. Lambek calculus is NP-complete. TCS, 357(1-3):186-201, 2006.

[33] D. Pous. Web appendix for 34 and this paper, http://perso.ens-lyon.fr/damien.pous/utas.

[34] D. Pous. Untyping typed algebraic structures and colouring proof nets of cyclic linear logic. In Proc. $C S L$, volume 6247 of $L N C S$, pages 484-498. Springer, August 2010.

[35] V. R. Pratt. Action logic and pure induction. In Proc. JELIA, volume 478 of LNCS, pages 97-120. Springer, 1990.

[36] V. Redko. On defining relations for the algebra of regular events (Russian). Ukrain. Mat. Z., 16:120-126, 1964.

[37] L. Regnier. Lambda-calcul et réseaux. Thèse de doctorat, Univ. Paris VII, 1992.

[38] V. Siles and H. Herbelin. Equality is typable in semi-full pure type systems. In Proc. LICS, pages 21-30. IEEE, 2010.

[39] A. Wille. A Gentzen system for involutive residuated lattices. Alg. Univ., 54:449-463, 2005.

[40] D. Yetter. Quantales and (noncommutative) linear logic. J. Sym. Log., 55(1):41-64, 1990.

This work is licensed under the Creative Commons Attribution-NoDerivs License. To view a copy of this license, visit http://creativecommons.org/licenses/by-nd/2.0/ or send a letter to Creative Commons, 171 Second St, Suite 300, San Francisco, CA 94105, USA, or Eisenacher Strasse 2, 10777 Berlin, Germany 